\title{
A Compact Retinal-Surgery Telemanipulator that Uses Disposable Instruments
}

\author{
Manikantan Nambi ${ }^{1}$, Paul S. Bernstein ${ }^{2}$, and Jake J. Abbott ${ }^{1}$ \\ 1 Department of Mechanical Engineering \\ 2 Department of Ophthalmology and Visual Science, Moran Eye Center \\ University of Utah \\ Salt Lake City, UT, 84112, USA \\ \{m.nambi,jake.abbott\}@utah.edu, paul.bernstein@hsc.utah.edu
}

\begin{abstract}
We present a retinal-surgery telemanipulation system with submicron precision that is compact enough to be head-mounted and that uses a full range of existing disposable instruments. Two actuation mechanisms are described that enable the use of actuated instruments, and an instrument adapter enables quick-change of instruments. Experiments on a phantom eye show that telemanipulated surgery results in reduction of maximum downward force on the retina as compared to manual surgery for experienced users.
\end{abstract}

\section{Introduction}

Retinal microsurgery procedures are at the limits of human ability [1]. An error of only a few micrometers can cause the instrument to exert damaging force on the retina, causing loss of vision at the spot. The forces experienced during retinal surgeries are below what surgeons can feel, so surgeons must rely on visual feedback only [2]. The surgeon must pivot the instruments about the scleral trocars, limiting dexterity, and must use the instruments to manipulate the eye to provide better imaging through the surgical microscope. Patient movement due to breathing must be accounted for by the surgeon, and in addition, among patients who snore under monitored anesthesia ( $\approx 16 \%$ of cases [3]), half have sudden head movements during surgery, leading to a high risk of complications.

One of the most difficult retinal-surgery procedures involves the peeling of membranes on the retina. Epiretinal membranes (ERM), sheets of fibrous tissue up to $61-\mu \mathrm{m}$-thick [4] that distort macular anatomy and disturb vision after posterior vitreous detachment or retinal tears, and the inner limiting membrane, a naturally occurring $0.15-4-\mu \mathrm{m}$-thick membrane [5] that can contract with age and generate macular holes, are peeled to improve vision in affected eyes. Membrane peeling is a delicate procedure, and complications occur in the form of intraoperative hemorrhage, retinal detachment during or after surgery, regrowth of ERM, and increased rate of cataract development [6. In some cases, a second surgery is required to remove fragments of the ERM left behind. There are opportunities for significant improvement in this and other retinal-surgery procedures in terms of safety and consistency of outcomes. Robot-assisted retinal 
surgery will enable surgeons to overcome their human limitations, and to extend their working life even after their manual abilities have diminished.

Prior research in robot-assisted retinal surgery has resulted in development of telemanipulated systems [7-11], co-operative manipulators [12], and active handheld instruments [13. The robotic systems have typically been relatively large and stiff, and thus table-mounted. The hand-held device is a clear exception, but it is primarily aimed at tremor reduction, with no ability to affect the "DC" system response. Most prior systems leave the retina at risk in the event of sudden head movement, and rhythmic head movements would need to be actively compensated. Notable exceptions are the TU Munich [10] and Columbia/Vanderbilt systems [8], which are designed to be head-mountable.

The specifications of retinal surgery are difficult to achieve using traditional mechatronic components (e.g., motors, gears), while maintaining a small form factor. In this paper, we present a manipulator for retinal surgery that utilizes piezoelectric stick-slip actuators, which were designed specifically for micromanipulation (this same style of actuator was used in [10]). The result is a manipulator with submicron resolution that is small and light enough to be head-mounted. A principal contribution of this work is an instrument adapter that enables the use of the full range of existing disposable actuated (microforceps, scissors) and non-actuated (diamond-dusted scraper (DDS), vitrector, fibre-optic light) instruments, and enables quick change of instruments, which is an important requirement in retinal surgery that has never been demonstrated in any of the prior telemanipulated systems. We also describe a custom master input device that mimics a disposable microforceps. Finally, we include some preliminary experimental demonstrations of membrane peeling in a force-sensitive phantom eye. Our complete system is shown in Fig. 1.

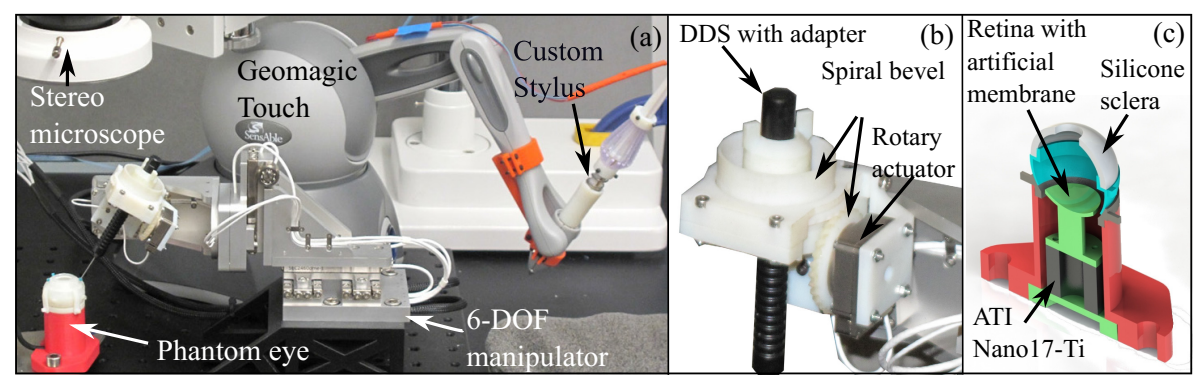

Fig. 1. Experimental setup of the retinal-surgery system. The surgeon looks in the phantom eye using a stereo microscope, and telemanipulates the end-effector of the instrument with 4-DOF (3-DOF translation, and rotation of the instrument about its axis) using a Geomagic Touch (located to enable direct access to instruments) with a custom stylus that is constrained to have the same 4-DOF by locking the wrist. (b) Yaw joint of the manipulator, which is responsible for rotation of the instrument about its axis, with an adapter that enables instruments to be attached to the manipulator. (c) Phantom eye used in experiments. 


\section{System Design}

A six-degree-of-freedom (6-DOF) manipulator was designed using off-the-shelf piezoelectric stick-slip actuators from SmarAct GmbH (Fig. 1). It comprises a 3 -DOF translation stage and a 3-DOF spherical wrist, which enables the manipulator to position the instrument inside a $20-\mathrm{mm}$-diameter circle on the retina (positioning precision measured using joint sensors: $<1 \mu \mathrm{m}$, max. velocity: $6 \mathrm{~mm} / \mathrm{s}$ ) with a virtual remote center on the surface of the eye (a sphere of 25.4-mm diameter). The linear stages have a range of $40 \mathrm{~mm}$ with a closed-loop resolution of $100 \mathrm{~nm}$. The spherical wrist comprises three rotary actuators, with a closed-loop resolution of $25 \mu^{\circ}$ for the roll and pitch actuators, and with a yaw actuator that enables open-loop rotation about the axis of the instrument with a resolution of $3 \mathrm{~m}^{\circ}$. The manipulator measures $200 \times 100 \times 70 \mathrm{~mm}^{3}$ and weighs $0.8 \mathrm{~kg}$.

The manipulator was manufactured by SmarAct to our specifications, and we further modified the yaw joint of the manipulator such that it can use actuated and non-actuated instruments. The modified yaw joint was manufactured using a 3D printer (Objet Eden260). The yaw joint is designed with the yaw actuator's axis orthogonal to the instrument's axis, and the rotary motion to the instrument is transmitted using spiral bevel gears. The spiral bevel gear includes a 23-mm aperture and internal threads that enable instruments to be attached to the manipulator. The aperture size was selected such that disposable instruments of a wide range of form factors can be used with the manipulator.

From our observations in the operating room, we found that during retinal surgery, on average the surgeon changes the instrument every two minutes. It is important that a robotic system for such procedures facilitates the quick change of instruments without disturbing the flow of the procedure, so we designed an adapter that enables the surgeon to change instruments frequently, and enables the use of disposable instruments that require "pinch-grip" actuation such as microforceps and scissors, with this seventh DOF of actuation connected to the instrument rather than to the manipulator. Our mechanism utilizes adapters that are attached to disposable instruments before surgery; the adapter uses threads inspired by Luer fittings, such that the instrument can be inserted in the perfect position every time. Once the adapters are correctly affixed to the instruments (see Fig. 2 2 -e), the end-effector of any instrument will be at the same known location when inserted into the manipulator.

To characterize the instrument change time for our manipulator, we performed a simple experiment in which we changed the instrument from a DDS to a microforceps and then back to a DDS (5 trials), at a comfortable speed. The average time required to change an instrument was found to be $12 \mathrm{~s}$.

Two different actuation mechanisms were designed to enable the use of two different families of actuated instruments that are commonly used in retinal surgery. For actuating a disposable instrument tip (e.g., Synergetics micro-forceps tip (Fig. 2a)), which requires pressing a plunger on the device, we used a linear stepper motor (LC15, HaydonKerk) with force capability of $5 \mathrm{~N}(2 \mathrm{~N}$ is required to actuate a Synergetics microforceps). The stepper motor is attached to the microforceps tip using an adapter that enables the microforceps to be mounted 


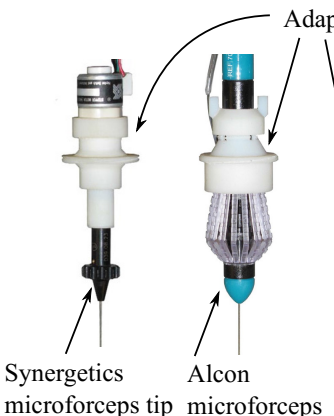

(a) (b)

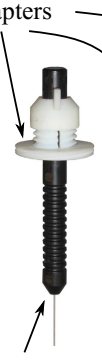
DDS

(c)

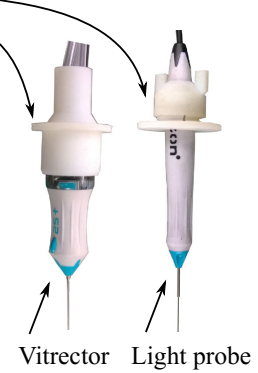

(d)

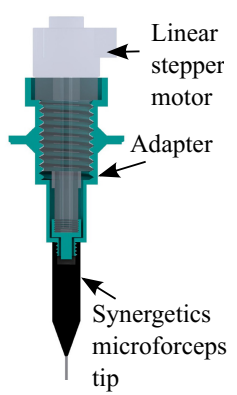

(f)

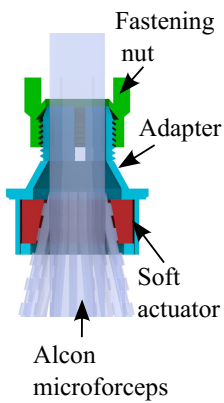

(g)

Fig. 2. (a)-(e) Disposable retinal-surgery instruments with adapters than enable quickchange mounting to the 6-DOF manipulator. (f) Section view of the Synergetics microforceps in (a) actuated by a linear stepper motor. (g) Section view of the Alcon microforceps in (b) actuated by a soft actuator.

on the manipulator (Fig. 2f). The LC15 has a linear resolution of $2.5 \mu \mathrm{m}$, and requires 500 steps (travel of $1.25 \mathrm{~mm}$ ) for the complete actuation of the microforceps. The measured bandwidth for a full open-close cycle of the microforceps with the stepper motor is $2.5 \mathrm{~Hz}$.

The second actuation mechanism, for use with completely disposable instruments (e.g., Alcon microforceps (Fig. 2 p , 2b)), comprises a soft actuator that is inspired by a blood-pressure cuff, which squeezes the ribs on a pinch-grip device when supplied with pressurized air (already available in the operating room). The soft actuator is molded from a silicone resin (Dragon Skin 20, Smooth-on Inc.) using soft-lithography techniques. A closed-loop control system comprising two ON/OFF valves (MHJ series, Festo) and a pressure sensor is implemented, and optimized for a bandwidth of $2 \mathrm{~Hz}$ and a resolution of 10 discrete steps between fully closed and fully open microforceps.

A Geomagic Touch haptic interface is modified with a custom stylus to telemanipulate the retinal manipulator (Fig. 1 a). The stylus is built to mimic an Alcon disposable microforceps, using components salvaged from its pinch-grip device. A linear potentiometer (ThinPot, Spectra Symbol) is used to measure the squeezing of the pinch-grip mechanism (resolution: $0.04 \mathrm{~mm}$, travel: $1.25 \mathrm{~mm}$ ), and a spring $(6 \mathrm{~N} / \mathrm{mm})$ recreates the stiffness of an actual microforceps.

A master-slave position controller (software-adjustable scaling, with a deadband of $200 \mu \mathrm{m}$ on the master) with a virtual spring-damper coupling between the master and slave positions is implemented. The gains were chosen to generate smooth and stable behavior. The remote-center-of-motion movement about the trocar is handled in software, such that the user only controls 4-DOF of instrument movement. Orbital manipulation is not implemented here, but nothing precludes it. In a telemanipulation experiment in which we attempted to generate the smallest possible instrument movement $(5$ trials in each of six cardinal directions), we measured, using joint sensors, a worst-case resolution of $38 \mu \mathrm{m}$ with $8: 1$ scaling, and $6 \mu \mathrm{m}$ with 100:1 scaling. 


\section{Membrane-Peeling Experiments and Results}

To compare manual vs. telemanipulated retinal surgery (using 8:1 scaling exclusively), we performed experiments with a phantom eye shown in Fig. 17. Trocars were inserted into the model eye as would be done in surgery. The anterior (upper portion) of the eye is made of a synthetic rubber (Phake-I, $8 \mathrm{~mm}$-diameter pupil), and approximates the size, shape, and feel of the human eye. The anterior of the eye was attached to a fixture as shown in Fig. 11, and inside the fixture, an ATI Nano17-Ti force/torque sensor (noise $<2 \mathrm{mN}$ ) was mounted with a section of a spherical surface that acts as the posterior (retinal) surface of the eye on which surgery will be performed. This mechanical isolation between the anterior and posterior of the eye ensures that only the relatively small instrument-retina interaction forces are measured by the force sensor. The retinal surface was prepared with an artificial membrane made of paper (cut to 6-mm-diameter circle, $120 \mu \mathrm{m}$ thickness), and $10 \mu \mathrm{l}$ of an eye lubricant gel (GenTeal) was used to achieve adhesion between the membrane and the model retina.

Three vitreoretinal surgeons with varying degrees of surgical experience20 years (expert), 2 years (intermediate), 6 months (novice) - and a graduate student with no experience in actual surgery, performed manual and telemanipulated surgery on the phantom eye setup with an Alcon microforceps. The graduate student and expert surgeon are both authors of this paper. All the surgeons had two hours of practice on the telemanipulated system before data was recorded. The graduate student had been using the telemanipulation system for a year. Two experiments were performed by each subject. In experiment 1 , subjects performed manual surgery, and in experiment 2 the surgery was performed with the telemanipulated system. The subjects had to completely peel a membrane off the retina with the microforceps. Six trials were performed in each experiment, spread across two days. Two subjects (expert and novice) performed experiment 1 followed by experiment 2 on the first day, with the order reversed on the second day, and the other subjects (intermediate and graduate student) performed the experiments in a reverse order. A fresh membrane was prepared for each trial. Although we do not purport that this pilot study is a rigorous experiment on which we can make strong claims, we do believe that the results are informative about the potential of the telemanipulated system.

To evaluate performance in our experiments, we use the maximum downward force $\left(F_{-z}\right)$ and the completion time $\left(T_{c}\right)$ in a trial as metrics; Fig. 3 3 shows data for all subjects and trials. The stiffness of the retina used in our experiments is higher than that of an actual retina, and hence, the forces measured can only be used for comparisons within this study. The first result we observe in the data is that the expert surgeon improves significantly from Day 1 to Day 2 with the telemanipulated system $(F(1,4)=7.5, p=0.05)$, bringing his force level down to approximately that of his manual surgery.

Next, we observe that all four subjects perform approximately equivalently during manual surgery in terms of force, and that the expert and intermediate surgeons (which we will refer to as the skilled surgeons) perform substantially better than the other two subjects during manual surgery in terms of time. We 
also observe there are no noticeable trends (e.g., learning) from Day 1 to 2 for manual surgery, as we would expect. As a result, for all subsequent analysis we lump the two days of manual data together for a given subject to increase the power of the statistics. In addition, we lump the two days of manual data for the expert and intermediate surgeons into a single skilled manual data set.

Next, we observe that for the graduate student, who is an expert user with the telemanipulation system, forces are lower in telemanipulated surgery (with Days 1 and 2 lumped together) than in manual surgery $(F(1,10)=10.9, p=$ 0.008); however, his completion time may be slightly slower. We also find that his telemanipulated forces are lower than those of the skilled surgeons' manual forces $(F(1,16)=11.9, p=0.003)$; however, his completion time is longer $(F(1,16)=40.2 ; p<0.001)$.

Similarly, but maybe more promising, for the novice surgeon with limited surgical experience, forces are lower in telemanipulated surgery on Day 2 than in manual surgery $(F(1,7)=3.9, p=0.094)$; in addition, his completion time on Day 2 may be slightly shorter than in manual surgery. We also find that the novice surgeon's telemanipulated forces on Day 2 are lower than those of the skilled surgeons' manual forces $(F(1,13)=11.6, p=0.005)$; however, his completion time is longer $(F(1,13)=38.5, p<0.001)$.

Finally, we observed that the high positioning resolution in telemanipulated surgery sometimes resulted in the membrane being peeled off in layers, and multiple grasping actions were required to peel the membrane, which contributed to a higher $T_{c}$. It may be necessary to train users of the telemanipulator to penetrate deep enough into the retina to grasp the entire membrane.

\section{Discussion}

In terms the achievable precision and velocity at the instrument's end-effector, our manipulator compares well with other retinal-surgery manipulators (Table1).
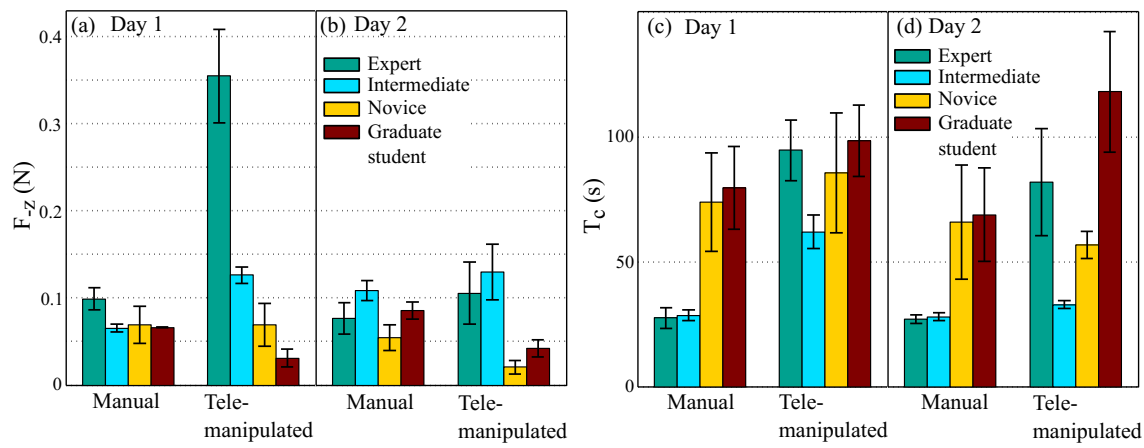

Fig. 3. Experimental results comparing manual and telemanipulated membrane peeling with a microforceps. (a)-(b) shows maximum downward force $\left(F_{-z}\right)$, and (c)-(d) shows completion time $\left(T_{c}\right)$. Data is divided according to subject, day, and mode of experiment. Error bars indicate standard deviation. 
Table 1. Comparison of robot-assisted retinal-surgery systems.

\begin{tabular}{|c|c|c|c|c|}
\hline System & Precision & Velocity & $\begin{array}{c}\text { Head- } \\
\text { mountable }\end{array}$ & $\begin{array}{c}\text { Quick-change/existing } \\
\text { actuated instruments }\end{array}$ \\
\hline Northwestern [7] & $0.2 \mu \mathrm{m}$ & NA & No & No/No \\
\hline Johns Hopkins [12] & $<1 \mu \mathrm{m}$ & $5 \mathrm{~mm} / \mathrm{s}$ & No & Yes/No \\
\hline Univ. of Tokyo [9] & $5 \mu \mathrm{m}$ & NA & No & No/Yes \\
\hline TU Eindhovan [1] & $10 \mu \mathrm{m}$ & NA & No & Yes/No \\
\hline Columbia/Vanderbilt [8] & $<5 \mu \mathrm{m}$ & NA & Yes & No/Yes \\
\hline TU Munich [10] & $5 \mu \mathrm{m}$ & $40 \mathrm{~mm} / \mathrm{s}$ & Yes & NA/NA \\
\hline Our System & $<\mathbf{1} \mu \mathbf{m}$ & $\mathbf{6 ~} \mathbf{m m} / \mathbf{s}$ & Yes & Yes/Yes \\
\hline
\end{tabular}

During actual membrane peeling, instrument velocities have been measured in the range of $0.1-0.5 \mathrm{~mm} / \mathrm{s}$ [14, which our manipulator is easily capable of achieving. However, during repositioning tasks, velocities higher than our maximum of $6 \mathrm{~mm} / \mathrm{s}$ would be desirable, if the goal is to recreate instrument movements similar to manual surgery. Different kinematics could be used to modify the resolutionvelocity trade-off. Regardless, the quick-change adapter, disposable-instrument actuators, and custom stylus presented here could be utilized with any manipulator kinematics, including existing manipulators (Table1). Our system could also incorporate force-sensing instruments [14] for improved safety.

Due to the underactuation of our inexpensive haptic interface (6-DOF with only 3 -DOF actuation), we constrained our haptic interface to have the same 4-DOF as the instrument by locking the wrist angle of the haptic stylus. Also, because of the fixed trocar point in telemanipulated surgery, orbital movement of the eye was not possible. As a result, the hand motions required in telemanipulated surgery with our haptic interface was fundamentally different than in manual surgery. The two subjects who perform better than manual surgery with the telemanipulated system also have the least experience in real surgery. Moving to a haptic master that more closely matches hand motions observed in real surgery may be important for improving performance with the system, and for enabling intuitive orbital manipulation.

In our experiments, subjects manually manipulated a light-probe in the phantom eye with their left hand while telemanipulating the instrument with their right hand. This leads to bending of delicate instruments when both instruments do not work in concert, resulting in unintended motion at the end-effector. To truly demonstrate the precision capabilities of the telemanipulated system, all manual interaction should be removed by telemanipulating both instruments. Additionally, we expect that a phantom that mimics human head/eye movement during surgery would highlight the benefits of a head-mounted manipulator.

Acknowledgements. This project was funded by two Intuitive Surgical Technology Research Grants. 


\section{References}

1. Riviere, C.N., Rader, R.S., Khosla, P.K.: Characteristics of hand motion of eye surgeons. In: Int. Conf. IEEE. Engineering in Medicine and Biology Society, pp. 1690-1693 (1997)

2. Gupta, P.K., Jensen, P.S., de Juan Jr., E.: Surgical forces and tactile perception during retinal microsurgery. In: Taylor, C., Colchester, A. (eds.) MICCAI 1999. LNCS, vol. 1679, pp. 1218-1225. Springer, Heidelberg (1999)

3. McCannel, C.A., Olson, E.J., Donaldson, M.J., Bakri, S.J., Pulido, J.S., Mueller, D.: Snoring is associated with unexpected patient head movement during monitored anesthesia care vitreoretinal surgery. Retina 32(7), 1324-1327 (2012)

4. Wilkins, J.R., Puliafito, C.A., Hee, M.R., Duker, J.S., Reichel, E., Coker, J.G., Schuman, J.S., Swanson, E.A., Fujimoto, J.G.: Characterization of epiretinal membranes using optical coherence tomography. Ophthalmology 103(12), 2142-2151 (1996)

5. Henrich, P.B., Monnier, C.A., Loparic, M., Cattin, P.C.: Material properties of the internal limiting membrane and their significance in chromovitrectomy. Ophthalmologica 230(2), 11-20 (2013)

6. Donati, G., Kapetanios, A.D., Pournaras, C.J.: Complications of surgery for epiretinal membranes. Graefe's Archive for Clinical and Experimental Ophthalmology 236(10), 739-746 (1998)

7. Jensen, P.S., Grace, K.W., Attariwala, R., Colgate, J.E., Glucksberg, M.R.: Toward robot-assisted vascular microsurgery in the retina. Graefe's Archive for Clinical and Experimental Ophthalmology 235(11), 696-701 (1997)

8. Wei, W., Popplewell, C., Chang, S., Fine, H.F., Simaan, N.: Enabling technology for microvascular stenting in ophthalmic surgery. J. Medical Devices 4(1), 14503 (2010)

9. Ida, Y., Sugita, N., Ueta, T., Tamaki, Y., Tanimoto, K., Mitsuishi, M.: Microsurgical robotic system for vitreoretinal surgery. Int. J. Computer Assisted Radiology and Surgery 7(1), 27-34 (2012)

10. Nasseri, M., Eder, M., Nair, S., Dean, E., Maier, M., Zapp, D., Lohmann, C., Knoll, A.: The introduction of a new robot for assistance in ophthalmic surgery. In: Int. Conf. IEEE Engineering in Medicine and Biology Society, pp. 5682-5685 (2013)

11. Meenink, H., Hendrix, R., Naus, G., Beelen, M., Nijmeijer, H., Steinbuch, M., Oosterhout, E., Smet, M.: Robot-assisted vitreoretinal surgery. In: Medical Robotics: Minimally Invasive Surgery, pp. 185-209 (2012)

12. Uneri, A., Balicki, M.A., Handa, J., Gehlbach, P., Taylor, R.H., Iordachita, I.: New steady-hand eye robot with micro-force sensing for vitreoretinal surgery. In: IEEE Int. Conf. Biomedical Robotics and Biomechatronics, pp. 814-819 (2010)

13. MacLachlan, R.A., Becker, B.C., Cuevas Tabarés, J., Podnar, G.W., Lobes, L.A., Riviere, C.N.: Micron: an actively stabilized handheld tool for microsurgery. IEEE Trans. Robotics 28(1), 195-212 (2012)

14. Balicki, M., Uneri, A., Iordachita, I., Handa, J., Gehlbach, P., Taylor, R.: Microforce sensing in robot assisted membrane peeling for vitreoretinal surgery. In: Jiang, T., Navab, N., Pluim, J.P.W., Viergever, M.A. (eds.) MICCAI 2010, Part III. LNCS, vol. 6363, pp. 303-310. Springer, Heidelberg (2010) 